\title{
Simultaneous Registration and Intensity Normalization of SPECT Perfusion Images
}

\author{
Margarida Silveira and Pedro M. Q. Aguiar \\ Institute for Systems and Robotics / IST
}

\begin{abstract}
The analysis of 3D SPECT brain images requires several pre-processing steps such as registration, intensity normalization and brain extraction. Usually, registration is performed before intensity normalization, which requires robust registration methods, such as those based on the maximization of the Mutual Information (MI), which are computationally complex. In this paper we propose using a computationally simple method to perform the simultaneous registration and intensity normalization of SPECT brain perfusion images. The approach, which extends to 3D data a method originally proposed in [1] for 2D photographic images, estimates in alternate steps the intensity normalization parameters and the registration parameters. Our experiments, with real SPECT images, show that the proposed registration method leads to results similar to those obtained by using more expensive algorithms such as those based on the MI criterion.
\end{abstract}

Index Terms-medical image registration, brain SPECT, intensity normalization

\section{INTRODUCTION}

Cerebral perfusion images acquired by single photon emitting computer tomography (SPECT) provide functional rather than anatomical information. They are commonly used for the diagnostic of dementias such as Alzheimer's Disease, Parkinson's Disease and Dementia with Lewy bodies. An important pre-processing step when dealing with these images is their registration, or alignment. The challenge of this step is due to the characteristics of the three-dimensional (3D) images to be registered: they suffer from poor spatial resolution and contrast (see an example in Fig. 1). For this kind of images, feature-based image registration techniques are clearly not appropriate.

Also, in what concerns common intensity-based techniques, a direct comparison between the SPECT image intensities is not possible. In fact, take the example of the Tc-99m HMPAO, a typical tracer used in SPECT imaging. The generated volumes give a blood flow measure that is relative to the blood flow in other regions of the brain. Therefore, registering by directly comparing voxel intensities does not make sense, even if the images correspond to different acquisitions of the same subject.

To deal with the difficulty just outlined, complex algorithms based on registration criteria like the Correlation Ratio (CR) [2] or the Mutual Information (MI) [3], which are very popular for intra modal registration, are usually adopted. In these cases, intensity normalization is performed after registration. For instance, in [4] registration is performed with the MI criterion followed by intensity normalization, assuming a linear model and using the least squares criterion. In [5], the
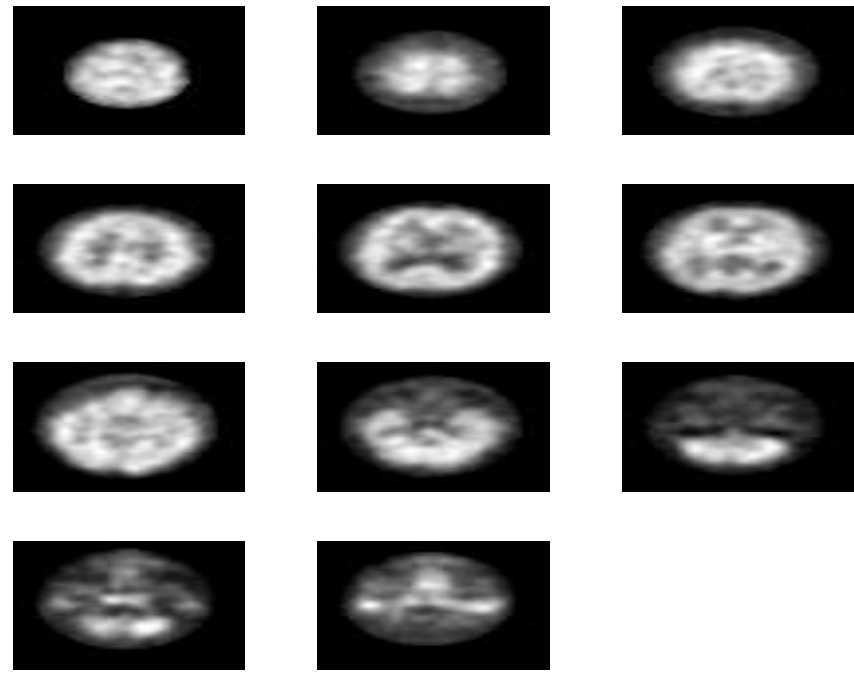

Fig. 1. Slices of a SPECT series.

registration criterion is the $\mathrm{CR}$, which is maximized using Powell optimization, and intensities are normalized by using an affine transformation. Other approaches attempt to remove the intensity inconsistencies with a preprocessing step, e.g., [6], and then perform the registration with simpler algorithms based on the least squares criterion [7].

In this paper we propose using a computationally simple method to perform registration and intensity normalization simultaneously. This method is an extension to $3 \mathrm{D}$ of the one proposed in [1] to deal with 2D photos. In our method, registration and normalization parameters are jointly estimated by using a two-step iterative, i.e., alternate, algorithm. Both steps result computationally simple: the solution optimizing the normalization parameters is obtained in closed form; and the solution optimizing the registration parameters corresponds to a 3D version of the usual intensity-based registration techniques. In this paper, we compare our registration algorithm with the one most widely used by the medical imaging community, which is based on the maximization of the MI, concluding on its effectiveness.

The remainder of the paper is organized as follows: Section II presents our method, Section III describes data and experiments, and Section IV concludes the paper.

\section{REGISTRATION AND INTENSITY NORMALIZATION}

In this section we describe the proposed method to perform the simultaneous registration and intensity normalization of 
the 3D SPECT images and briefly outline the competing approach based on maximization of MI.

\section{A. Problem formulation}

To state the problem, let $I_{1}(x, y, z)$ and $I_{2}(x, y, z)$ denote the two volumetric images to be registered. We assume that the image intensity levels are related by the following model:

$$
I_{1}(x, y, z)=\alpha I_{2}(W(\theta ; x, y, z))+\beta \text {, }
$$

where $\alpha$ and $\beta$ are the intensity normalization parameters - they represent the scale and offset of the intensity transformation - and $\theta$ is a vector collecting the registration parameters defining the geometric transformation $W$. In our case, this geometric transformation is assumed to be 3D affine, thus parameterized by a set of 12 parameters, i.e., $\theta=\left[\theta_{1}, \theta_{2}, \theta_{3}, \theta_{4}, \theta_{5}, \theta_{6}, \theta_{7}, \theta_{8}, \theta_{9}, t_{x}, t_{y}, t_{z}\right]^{T}$ and $W$ is defined by

$$
W(\theta ; x, y, z)=\left[\begin{array}{ccc}
\theta_{1} & \theta_{2} & \theta_{3} \\
\theta_{4} & \theta_{5} & \theta_{6} \\
\theta_{7} & \theta_{8} & \theta_{9}
\end{array}\right]\left[\begin{array}{l}
x \\
y \\
z
\end{array}\right]+\left[\begin{array}{l}
t_{x} \\
t_{y} \\
t_{z}
\end{array}\right] .
$$

Our goal when aligning, or registering, the 3D images $I_{1}(x, y, z)$ and $I_{2}(x, y, z)$ is to jointly estimate the parameters $\theta, \alpha$ and $\beta$. That leads to the joint minimization of a cost function $E(\theta, \alpha, \beta)$ that will be the usual sum of the squared errors:

$$
\begin{gathered}
\{\hat{\theta}, \hat{\alpha}, \hat{\beta}\}=\arg \min _{\theta, \alpha, \beta} E(\theta, \alpha, \beta), \\
E(\theta, \alpha, \beta)=\sum_{x, y, z} e^{2}(\theta, \alpha, \beta), \\
e(\theta, \alpha, \beta)=I_{1}(x, y, z)-\alpha I_{2}(W(\theta ; x, y, z))-\beta .
\end{gathered}
$$

To minimize (3), we use a two-step iterative method. In one of the steps, $\theta$ is kept fixed and (4) is minimized with respect to $\alpha$ and $\beta$. In the other one, $\alpha$ and $\beta$ are kept fixed and (4) is minimized with respect to $\theta$. As it will be seen in the sequel, the first step exploits the fact that the error (5) is linear in the intensity unknowns $\alpha$ and $\beta$. The second step leads to a 3D version of intensity-based registration.

\section{B. Estimation of the intensity normalization parameters}

Since the local errors in (5) are linear in the unknown parameters, the minimization of their sum in (3) is obtained in closed-form. In fact, the estimates $\hat{\alpha}$ and $\hat{\beta}$ are simply given by

$$
\begin{gathered}
\hat{\alpha}=\frac{N \sum_{x, y, z} I_{1} I_{2}-\sum_{x, y, z} I_{1} \sum_{x, y, z} I_{2}}{N \sum_{x, y, z} I_{2}^{2}-\left(\sum_{x, y, z} I_{2}\right)^{2},} \\
\hat{\beta}=\frac{\sum_{x, y, z} I_{1} \sum_{x, y, z} I_{2}^{2}-\sum_{x, y, z} I_{1} \sum_{x, y, z} I_{1} I_{2}}{N \sum_{x, y, z} I_{2}^{2}-\left(\sum_{x, y, z} I_{2}\right)^{2}},
\end{gathered}
$$

where $N$ is the number of voxels in the 3D region of summation of the cost $e^{2}(\theta, \alpha, \beta)$ in (4). In $(6,7)$, the arguments of $I_{1}$ and $I_{2}$ were omitted, for simplicity, i.e., $I_{1}$ stands for $I_{1}(x, y, z)$ and $I_{2}$ for $I_{2}(W(\theta ; x, y, z))$, see [1] for details.

\section{Estimation of the registration parameters}

Now $\alpha$ and $\beta$ are kept fixed and (4) is minimized with respect to $\theta$. This is done in an efficient way by developing a 3D version of the Lucas Kanade algorithm [8] (see also a recent review in [9]).

Our algorithm for this step is then iterative: the estimate $\hat{\theta}$ is found by updating a previous guess $\theta_{0}$, i.e., $\hat{\theta}=\theta_{0}+\Delta \theta$, where $\Delta \theta$ is found by minimizing (4) with respect to $\theta=$ $\theta_{0}+\Delta \theta$. Although this problem is nonlinear, a Gauss-Newton approach, where the local errors are approximated by their first-order truncated Taylor series expansion, provides a good linear approximation.

In our case, this linearization is equivalent to considering

$$
I_{2}\left(W\left(\theta_{0}+\Delta \theta\right)\right) \simeq I_{2}\left(W\left(\theta_{0}\right)\right)+\nabla I_{2} \nabla W \Delta \theta,
$$

where $\nabla I_{2}$ is the spatial gradient of the volumetric image $I_{2}$, evaluated at $W\left(\theta_{0}\right)$, and $\nabla W$ is the gradient of the geometric transformation $W$ in (2) with respect to the registration parameters in $\theta$. Again, we omit the dependency in the volume coordinates $x, y, z$, for simplicity.

Using approximation (8) and making zero the gradient of the cost function (4) with respect to $\theta=\theta_{0}+\Delta \theta$, the update $\Delta \theta$ is obtained in closed-form as

$$
\Delta \theta=H^{-1} \sum_{x, y, z}\left(\nabla I_{2} \nabla W\right)^{T}\left(I_{1}-\alpha I_{2}\left(W\left(\theta_{0}\right)\right)-\beta\right),
$$

where the matrix $H$ is given by

$$
H=\sum_{x, y, z}\left(\nabla I_{2} \nabla W\right)\left(\nabla I_{2} \nabla W\right)^{T} .
$$

The computational simplicity of this step comes then from the fact that the local updates of the registration parameters are obtained in closed-form, as opposed to using computationally intensive approaches to compute the derivatives involved (e.g., by using perturbation analysis). Note also that both the image gradient $\nabla I_{2}$ and the derivatives in $\nabla W$, used in (9) and (10), and easily obtained from (2), are just computed once.

\section{Initialization}

The iterative method just outlined exhibits good convergence to the global minimum when the Taylor series approximation is valid. Since in practice we initialize the process by assuming the volumes are aligned, i.e., the intensity normalization parameters are initialized with $\alpha_{0}=1$ and $\beta_{0}=0$ and the geometric registration parameters are initialized with the identity mapping in (2), the process converges when the displacement between volumes is small.

To cope with large displacements, we use a multiresolution scheme, where the minimization is carried out in a coarse-to-fine manner. Basically, we build a pyramid of multiresolution volumes for $I_{1}(x, y, z)$ and $I_{2}(x, y, z)$. The 
algorithm starts by estimating the alignment parameters at the coarsest scale. Then, resolution is progressively increased, i.e., the parameter estimates obtained at a given resolution level are converted to the immediately finer level, providing the initialization for the algorithm at the new level. This coarse-to-fine estimation not only improves the convergence but it also reduces the computational cost, since the bulk of computation is done using very low resolution volumes.

\section{E. Maximization of the Mutual Information}

For completeness, we now outline a registration approach based on the maximization of the MI. This is the criterion most widely used to register medical images [10]. However, it is difficult to implement and has many parameters to tune.

The MI is defined by

$$
\operatorname{MI}\left(I_{1}, I_{2}\right)=h\left(I_{1}\right)+h\left(I_{2}\right)-h\left(I_{1}, I_{2}\right)
$$

where $h(\cdot)$ denotes the entropy of a random variable and $h(\cdot, \cdot)$ denotes the joint entropy of a pair of random variables. The maximization of the MI criterion in (11) requires the usage of computationally expensive ascent optimizers (in order to get precise results, the learning rate must be progressively reduced).

For this paper, we tested two different methods based on the maximization of the MI: the Viola-Wells method [3] and the Mattes method [11]. Both methods require estimating probability density functions in order to evaluate entropy. The first method estimates densities from the data with (Gaussian) Parzen windows; requiring thus the (hand-tuned) definition of the standard deviation of the Gaussian kernel. The second method estimates densities using histograms; therefore it also requires the (hand-tuned) definition of the number of bins. More details concerning these methods can be found in [12].

\section{EXPERIMENTAL RESULTS}

\section{A. Data}

Images of five subjects were acquired on a Millennium MG, a multi-geometry digital CSE (GE's patented) dual detector gamma camera. These detectors have a Useful Field of View (UFOV) of $36 \times 52 \mathrm{~cm}$ and a crystal thickness of $8.5 \mathrm{~mm}$. The image matrices were $128 \times 128$, on a $360 \hat{\mathrm{A}}^{\circ}$ orbit, with the smallest radius of rotation possible for each patient geometry, with both detectors activated and on a $180 \hat{\mathrm{A}}^{\circ}$ geometry, on a step and shoot mode, 64 projections/detector (total study corresponding to 128 projections), 30 seconds/projection. The exams were processed on a Xeleris Workstation, where iterative reconstruction was performed by filtered backprojection using a Butterworth filter. The exams were performed with the brain perfusion radiopharmaceutical stabilized HMPAO (hexamethyl propylene amine oxime) labeled with Techetium-99m. The minimum intensity value was 0 for all the volumes and the maximum ranged from 278 to 515 . Such a difference in intensities justifies the need for intensity normalization.

\section{B. Methods}

We tested the proposed method using multiresolution pyramids with 3 levels (trilinear interpolation was used to compute volume intensities at non-integer coordinates). For comparison, we performed MI-based registration by using the Insight Toolkit (ITK) software package [12], where the parameters were tuned to get the best possible performance (the best results were obtained with the Mattes implementation). The same 3-level multiresolution decomposition was used for this method.

\section{Accuracy}

In order to compare the accuracy of these two methods, for each of the 5 SPECT images, we synthesized 20 new volumes by applying random geometric and intensity transformations and adding noise. The geometric transformations corresponded to $3 \mathrm{D}$ translations within [-10, 10], scalings within $[0.9,1.1]$ and rotations about an arbitrary 3D axis of an angle within $[-\pi / 8, \pi / 8]$. The intensity transformations were like in model (1), with $\alpha \in[0.95,1.05]$ and $\beta \in$ $[-5,5]$. The additive noise is white Gaussian, with standard deviation 20. Then, we registered the synthesized volumes with the original ones, using both methods, and compared the estimates of the registration parameters with the ones used to generate the images. Table I collects the bias and the standard deviation of the estimates of each of the geometric transformation parameters, obtained by the two methods.

\begin{tabular}{c|cc|cc} 
& Proposed & & MI & \\
\hline & Bias & Std Dev & Bias & Std Dev \\
\hline$\theta_{1}$ & -0.0044 & 0.0427 & 0.0101 & 0.0113 \\
$\theta_{2}$ & 0.0072 & 0.0363 & -0.0278 & 0.2001 \\
$\theta_{3}$ & 0.0040 & 0.0229 & 0.0207 & 0.1715 \\
$\theta_{4}$ & 0.0015 & 0.0356 & 0.0369 & 0.1977 \\
$\theta_{5}$ & -0.0018 & 0.0402 & 0.0034 & 0.0071 \\
$\theta_{6}$ & 0.0035 & 0.0257 & 0.0223 & 0.1955 \\
$\theta_{7}$ & 0.0047 & 0.0415 & -0.0081 & 0.1716 \\
$\theta_{8}$ & -0.0013 & 0.0450 & -0.0176 & 0.1957 \\
$\theta_{9}$ & -0.0033 & 0.0203 & 0.0059 & 0.0118 \\
$t_{x}$ & 0.0522 & 1.9618 & -1.3045 & 1.5799 \\
$t_{y}$ & -0.3608 & 2.0009 & -1.3763 & 1.7902 \\
$t_{z}$ & -0.3845 & 1.1390 & -1.8496 & 2.2273
\end{tabular}

TABLE I

RESULTS OF 100 REGISTRATION TESTS USING RANDOM GEOMETRIC TRANSFORMATIONS AND A LINEAR INTENSITY TRANSFORMATION.

From Table I, we see that both methods perform well. A closer look shows that both the bias and standard deviations of the proposed method are smaller than those of the MI method. Naturally, this higher accuracy comes from the fact that the data generation was in accordance with the assumed model (1). We emphasize however that, in addition to better accuracy, the proposed method has lower computational cost and less parameters to tune.

\section{Sensitivity to model violation}

To test the robustness of our method to intensity transformations that violate the model assumed in (1), we synthesized volumes using and a non-linear intensity mapping 
$I_{1}=\alpha I_{2}^{\gamma}$, with $\gamma \in[0.6,1.5]$ ( $\alpha$ was set to keep the intensity values within range). Table II collects the results obtained by both methods, showing that the proposed method performed significantly better than the MI, both in terms of bias and standard deviation. These results show that the proposed method is robust, even when the intensity model is different from the assumed linear model.

\begin{tabular}{c|cc|cc} 
& Proposed & & MI & \\
\hline & Bias & Std Dev & Bias & Std Dev \\
\hline$\theta_{1}$ & -0.0006 & 0.0204 & 0.0322 & 0.0343 \\
$\theta_{2}$ & 0.0031 & 0.0101 & -0.1334 & 0.0256 \\
$\theta_{3}$ & 0.0038 & 0.0087 & -0.1380 & 0.0278 \\
$\theta_{4}$ & 0.0064 & 0.0130 & 0.1564 & 0.0135 \\
$\theta_{5}$ & 0.0032 & 0.0164 & 0.0194 & 0.0420 \\
$\theta_{6}$ & 0.0039 & 0.0071 & -0.3482 & 0.0516 \\
$\theta_{7}$ & 0.0073 & 0.0144 & 0.1768 & 0.0201 \\
$\theta_{8}$ & 0.0061 & 0.0137 & 0.3909 & 0.0262 \\
$\theta_{9}$ & -0.0016 & 0.0147 & 0.0425 & 0.0800 \\
$t_{x}$ & -0.3755 & 1.9601 & -4.1928 & 4.2728 \\
$t_{y}$ & -0.3792 & 1.7959 & -4.6680 & 4.5990 \\
$t_{z}$ & -0.4153 & 0.7476 & -1.5516 & 4.6535
\end{tabular}

TABLE II

RESULTS OF 20 REGISTRATION TESTS USING RANDOM GEOMETRIC TRANSFORMATIONS AND A NONLINEAR INTENSITY TRANSFORMATION.

\section{E. Illustration with two real volumes}

Finally, we used the proposed method to register two real 3D SPECT images of the brain, chosen at random from our data set. The images are shown in the top of Fig. 2, together with the result obtained after registration and intensity normalization using the proposed method. Using transparency, the rendering of the two SPECT exams is shown in the same image and their alignment is easily visualized. This example illustrates how the proposed method successfully aligned the original images. Naturally, a similar result can be obtained with the MI algorithm, at a much higher computational cost, provided the parameters are appropriately tuned.

\section{Conclusions}

We proposed a computationally simple method to perform the 3D registration and intensity normalization of brain SPECT perfusion images simultaneously. We compared this approach with the usual registration based on maximization of the Mutual Information and obtained even better performance. Although our experiments used brain SPECT data, our method seems also suited for the registration of other medical imaging modalities and other regions than the brain.

\section{ACKNOWLEDGMENTS}

This work was supported by Fundação para a Ciência e a Tecnologia (ISR/IST plurianual funding) through the POS Conhecimento Program that includes FEDER funds. The work of the second author was also partially supported by FCT grant MODI-PTDC/EEA-ACR/72201/2006. The authors would like to thank Dr. Ana Isabel Santos and Hospital Garcia de Orta for providing the images.

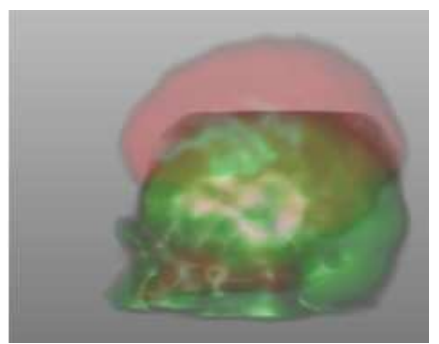

a)

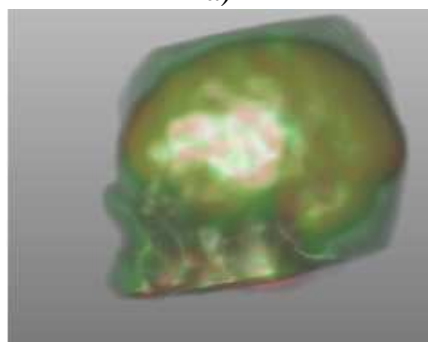

b)

Fig. 2. Rendering of the registration of two real 3D SPECT images using the proposed method: a) two images from the data set b) the same images after registration.

\section{REFERENCES}

[1] Aguiar, P., Unsupervised Simultaneous Registration and Exposure Correction, IEEE Int. Conf. on Image Processing, pp. 361-364, 2006.

[2] Roche, A., Malandain, G., Pennec, X. and Ayache, N., The Correlation Ratio as a New Similarity Measure for Multimodal Image Registration, Lecture Notes in Computer Science,Proc. MICCAI98, Vol. 1496, pp. 1115-1124, 1998.

[3] Viola, P. and Wells, W. M., Alignment by Maximization of Mutual Information, Int. Journal of Computer Vision, Vol. 24, no. 2, pp. 137154, 1997.

[4] Yuan-Lin Liao, Yung-Nien Sun, Nan-Tsing Chiu, Three dimensional registration and analysis for brain SPECT images, IEEE Nuclear Science Symposium Conference Record, Vol. 5, pp. 2715-2719, 2004.

[5] Stoeckel, J.; Fung, G., SVM feature selection for classification of SPECT images of Alzheimer's disease using spatial information, Fifth IEEE International Conference on Data Mining, pp. 8-12, 27-30 Nov. 2005.

[6] Imran, M.B., Mean regional cerebral blood flow images of normal subjects using technetium-99m-HMPAO by automated image registration, The Journal of nuclear medicine, vol. 39, no. 1, pp. 203 -207, 1998.

[7] Tang-Kai Yin, Nan-Tsing Chiu, Discrimination between Alzheimer's Dementia and Controls by Automated Analysis of Statistical Parametric Maps of 99mTc-HMPAO-SPECT Volumes, bibe, p. 183, Fourth IEEE Symposium on Bioinformatics and Bioengineering (BIBE'04), 2004.

[8] Lucas, B., Kanade, T., An iterative image registration technique with an application to stereo vision. Proceedings of the International Joint Conference on Artificial Intelligence, 1981.

[9] S. Baker, S. and Matthews, I., Lukas-kanade 20 years on: A unifiying framework, Int. Journal of Computer Vision, Vol. 56, no. 3, pp. 221255, 2004.

[10] Pluim, J.; Maintz, J. and Viergever, M., Mutual-information-based registration of medical images: a survey, IEEE Transactions on Medical Imaging, Vol. 22, no. 8, 2003.

[11] D. Mattes, D. R. Haynor, H. Vesselle, T. K. Lewellen, and W. Eubank. PET-CT image registration in the chest using free-form deformations. IEEE Trans. on Medical Imaging, Vol. 22, no. 1, January 2003.

[12] Ibanez, L.; Schroeder, W.; Ng,L. and Cates, J., ITK Software Guide, Kitware, Inc., 2005. 